Supporting Information

\title{
Light-Embrittled Dental Resin Cements Containing Photodegradable Polyrotaxane Cross-Linkers for Attenuating Debonding Strength
}

Satomi Matsunaga ${ }^{1}$, Atsushi Tamura ${ }^{2}$, Mayu Fushimi ${ }^{2}$, Hokuto Santa ${ }^{2}$, Yoshinori Arisaka ${ }^{2}$, Toru Nikaido ${ }^{3}$, Junji Tagami ${ }^{1}$, Nobuhiko Yui ${ }^{2, *}$

${ }^{1}$ Department of Cariology and Operative Dentistry, Graduate School of Medical and Dental Sciences, Tokyo Medical and Dental University (TMDU), 1-5-45 Yushima, Bunkyo, Tokyo 1138549, Japan

${ }^{2}$ Department of Organic Biomaterials, Institute of Biomaterials and Bioengineering, Tokyo Medical and Dental University (TMDU), 2-3-10 Kanda-Surugadai, Chiyoda, Tokyo 101-0062, Japan.

${ }^{3}$ Department of Operative Dentistry, Division of Oral Functional Science and Rehabilitation, School of Dentistry, Asahi University, 1851 Mizuho, Gifu 501-0296, Japan.

* Corresponding author: Nobuhiko Yui (E-mail address: yui.org@tmd.ac.jp)

\section{Table of Contents}

S1. Instrumentation

S2. Synthesis of bis(2-aminoethyl carbamate) poly(ethylene glycol) 


\section{S1. Instrumentation}

Fourier-transform infrared (FT-IR) spectra were recorded using a Spectrum 100 FTIR spectrometer (Perkin Elmer, Wellesley, MA, USA) equipped with an MCT (HgCdTe) detector. The samples were ground with $\mathrm{KBr}$ to prepare a pellet for transmission measurements. Two-dimensional nuclear Overhauser effect spectroscopy (2D NOESY) was performed using a Bruker Avance III $400 \mathrm{MHz}$ spectrometer in $\mathrm{NaOD} / \mathrm{D}_{2} \mathrm{O}$ at $25^{\circ} \mathrm{C} .2 \mathrm{D}$ NOESY was measured at the mixing time of $300 \mathrm{~ms}$.

\section{S2. Synthesis of bis(2-aminoethyl carbamate) poly(ethylene glycol)}

Poly(ethylene glycol) (PEG-OH, $M_{\mathrm{n}}=4,550$; Merck, Darmstadt, Germany) (20.0 g, 4.4 mmol) and 1,1'-carbonyldiimidazole (10.8 g, $66.7 \mathrm{mmol}$; Merck) were dissolved in tetrahydrofuran (THF; 240 $\mathrm{mL}$; Kanto Chemical, Tokyo, Japan) and allowed to react for $24 \mathrm{~h}$ at $25{ }^{\circ} \mathrm{C}$. After evaporating the solvent to dryness, the residue was dissolved in chloroform (Kanto Chemical). The solution was slowly added to diethyl ether (Kanto Chemical) to reprecipitate the polymer three times. The precipitate was collected by filtration and dried in vacuo to yield bis(imidazole carboxylate) PEG (PEG-IC 5k; $19.8 \mathrm{~g}$, $94.9 \%$ yield).

Next, PEG-IC 5k (19.8 g, $4.2 \mathrm{mmol})$ dissolved in THF (240 mL) was added dropwise (1 drop/sec) to the ethylenediamine (25 mL, $422.1 \mathrm{mmol}$; Fujifilm Wako Pure Chemical, Osaka, Japan) diluted with THF $(10 \mathrm{~mL})$, and the mixture was stirred for $24 \mathrm{~h}$ at $25^{\circ} \mathrm{C}$. After evaporating the solvent to dryness, the residue was dissolved in chloroform. The solution was slowly added to diethyl ether to reprecipitate the polymer three times. The precipitate was collected by filtration and dried in vacuo to yield bis(2-aminoethyl carbamate) PEG (PEG-NH2 5k; $16.4 \mathrm{~g}, 83.2 \%$ yield). 


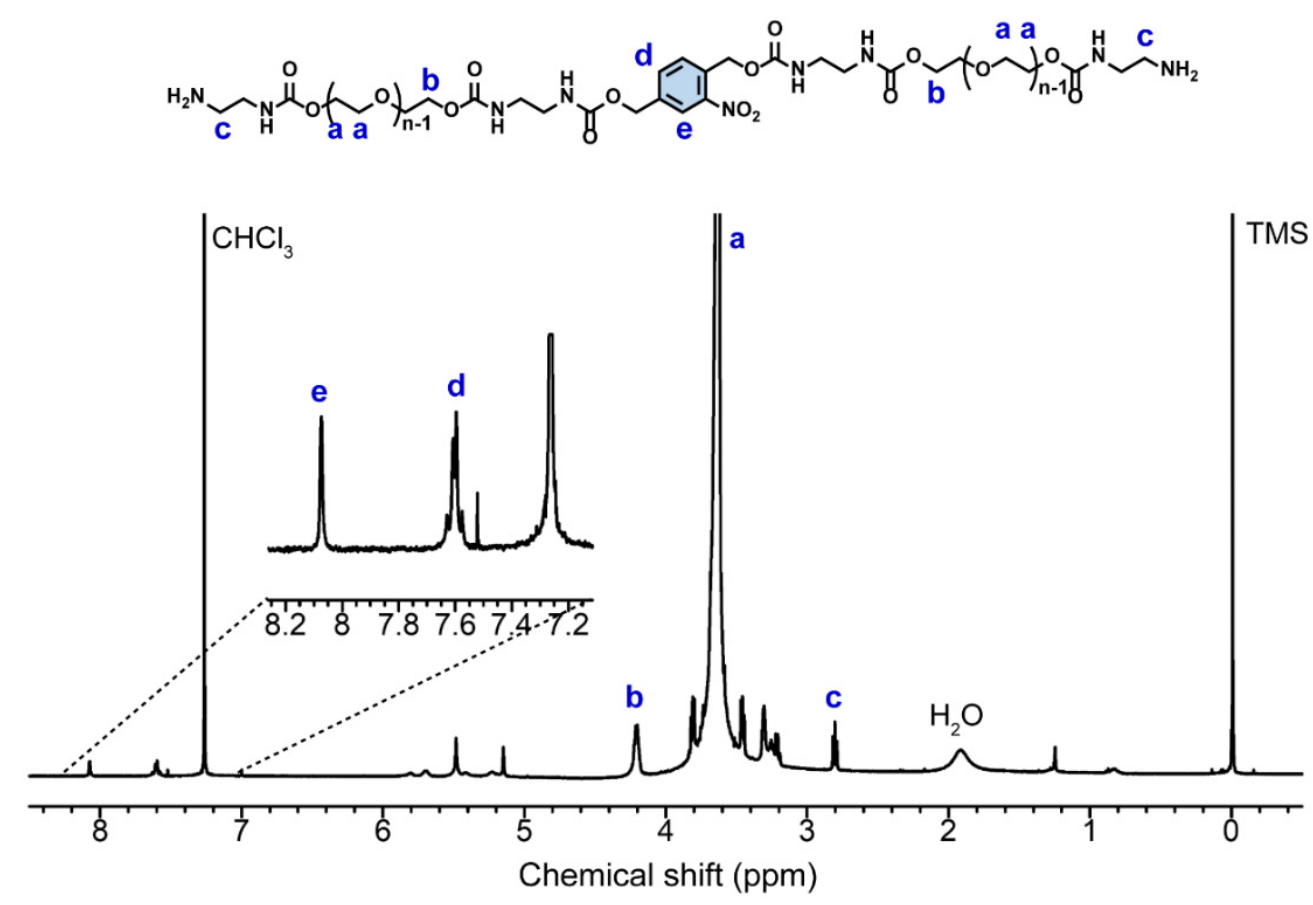

Figure S1. ${ }^{1} \mathrm{H}$ NMR spectrum of iNB-PEG in $\mathrm{CDCl}_{3}$ at $25^{\circ} \mathrm{C}$.

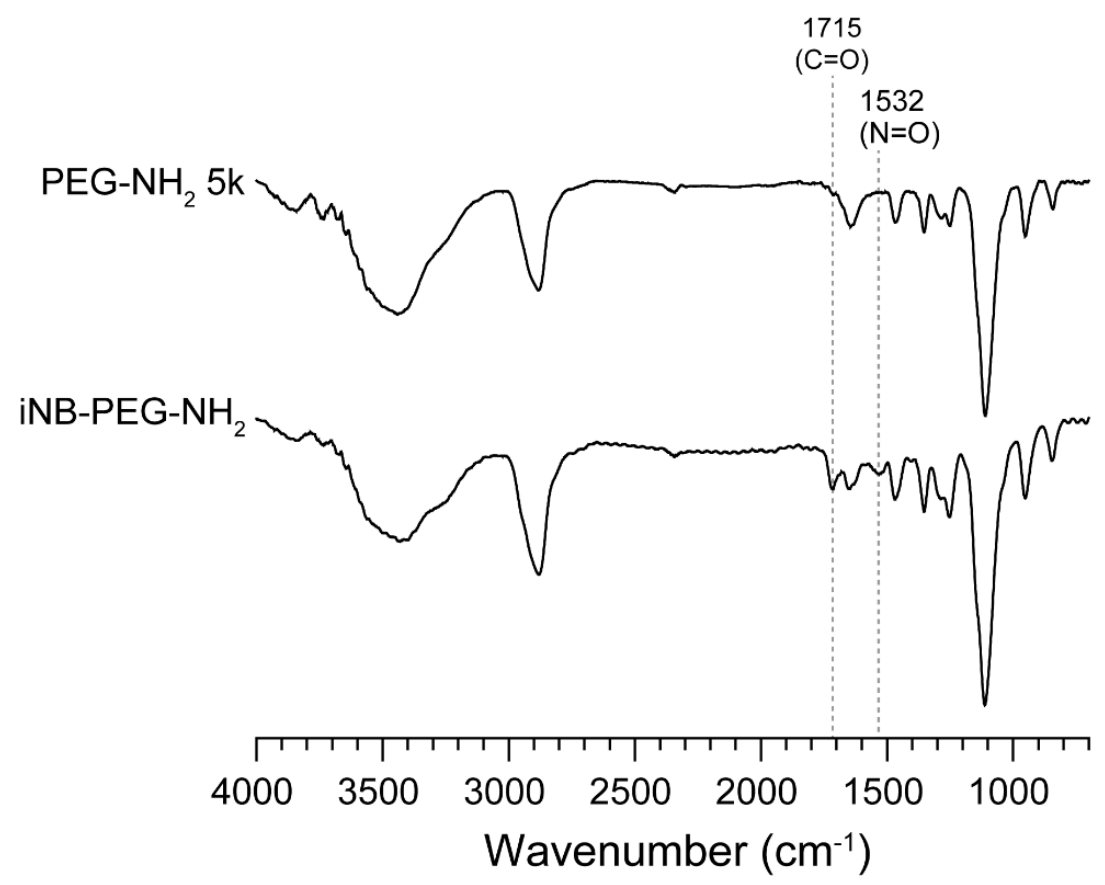

Figure S2. FT-IR spectra of PEG-NH2 $5 \mathrm{k}$ (upper) and iNB-PEG (lower). 

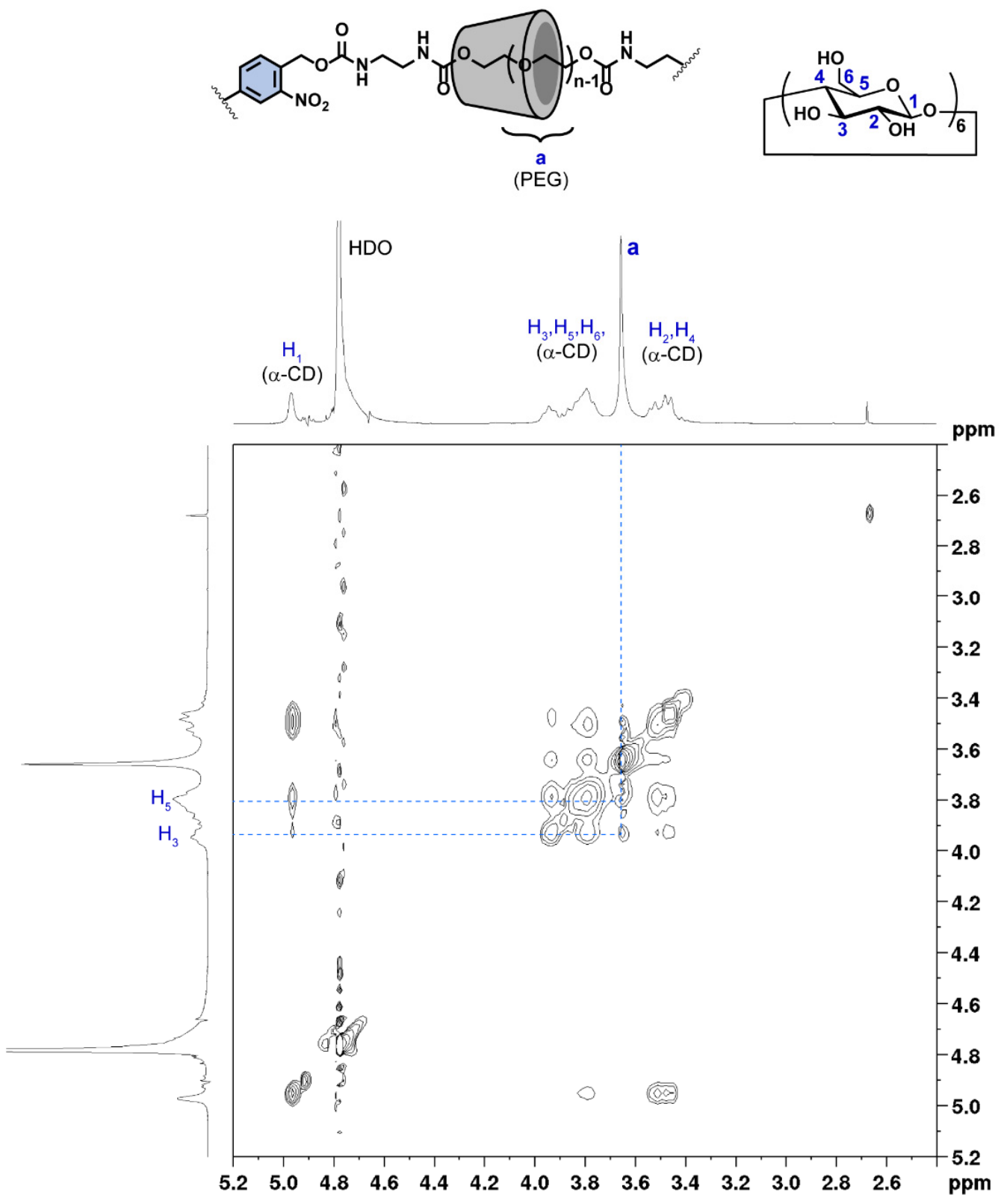

Figure S3. 2D NOESY spectrum of photodegradable iNB-PRX in $\mathrm{NaOD} / \mathrm{D}_{2} \mathrm{O}$ at $25^{\circ} \mathrm{C}$. 




Figure S4. FT-IR spectra of photodegradable iNB-PRX (upper) and MB-iNB-PRX (lower).

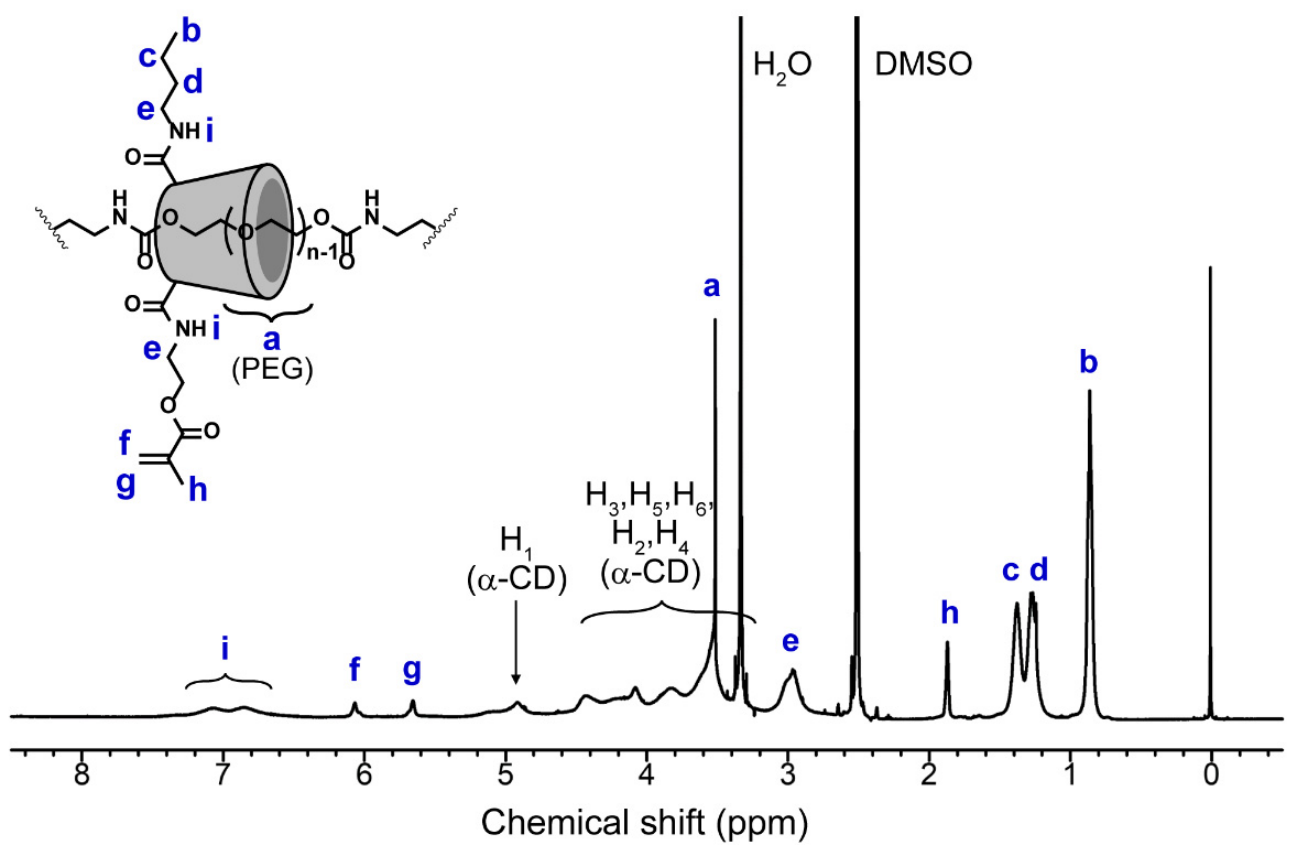

Figure S5. ${ }^{1} \mathrm{H}$ NMR spectrum of non-degradable MB-PRX in DMSO- $d 6$ at $25{ }^{\circ} \mathrm{C}$. 


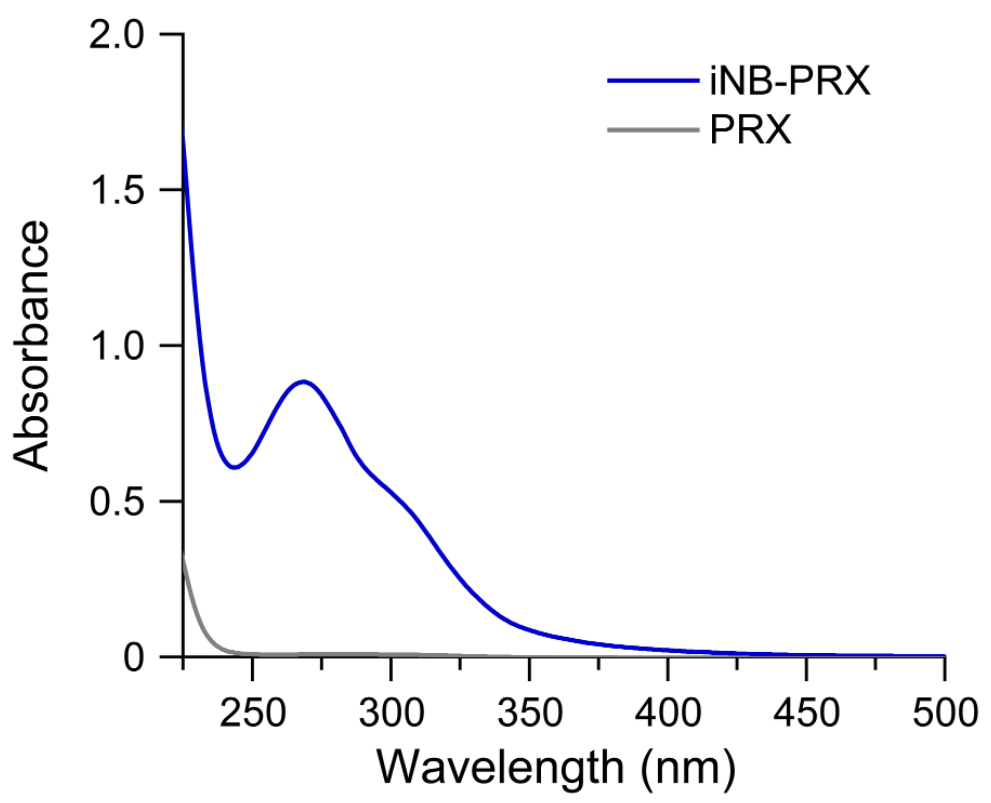

Figure S6. UV-vis absorption spectra of iNB-PRX (blue line) and non-degradable PRX (gray line) in $0.5 \mathrm{M} \mathrm{NaOH}$ solution $(10 \mathrm{mg} / \mathrm{mL})$.
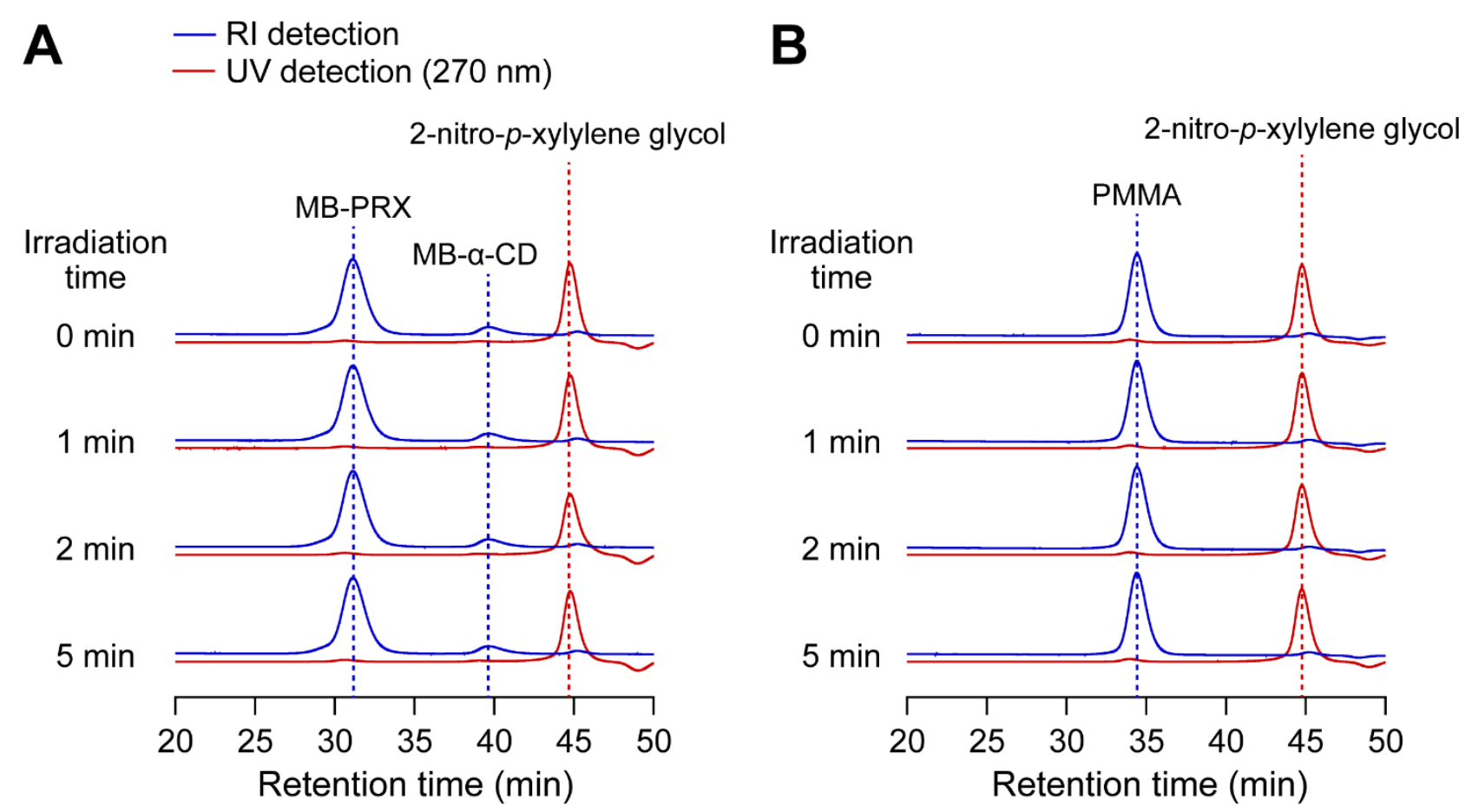

Figure S7. SEC charts of non-degradable MB-PRX (A) and PMMA $\left(M_{\mathrm{n}}=26,000\right)(\mathrm{B})$ containing 2nitro- $p$-xylylene glycol (1 wt\%) after UV irradiation for different times. The blue and red lines depict the chromatograms detected with refractive index (RI) and UV absorption at $270 \mathrm{~nm}$, respectively. 